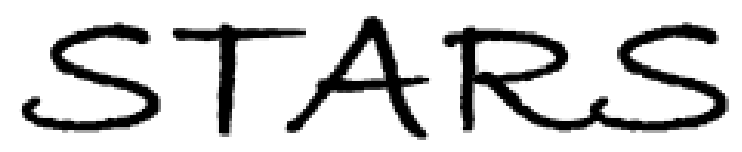

University of Central Florida

STARS

$1-1-2002$

\title{
Polymer depletion interaction between a colloid particle and a wall: A Monte Carlo study
}

Andrey Milchev

Aniket Bhattacharya

University of Central Florida

Find similar works at: https://stars.library.ucf.edu/facultybib2000

University of Central Florida Libraries http://library.ucf.edu

This Article is brought to you for free and open access by the Faculty Bibliography at STARS. It has been accepted for inclusion in Faculty Bibliography 2000 s by an authorized administrator of STARS. For more information, please contactSTARS@ucf.edu.

\section{Recommended Citation}

Milchev, Andrey and Bhattacharya, Aniket, "Polymer depletion interaction between a colloid particle and a wall: A Monte Carlo study" (2002). Faculty Bibliography 2000 s. 3365.

https://stars.library.ucf.edu/facultybib2000/3365

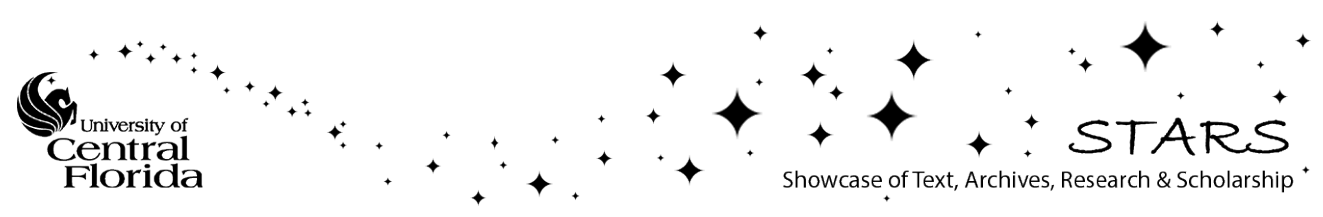




\section{Polymer depletion interaction between a colloid particle and a wall: A Monte Carlo study}

Cite as: J. Chem. Phys. 117, 5415 (2002); https://doi.org/10.1063/1.1499717

Submitted: 25 April 2002 . Accepted: 19 June 2002. Published Online: 27 August 2002

Andrey Milchev, and Aniket Bhattacharya

\section{ARTICLES YOU MAY BE INTERESTED IN}

On Interaction between Two Bodies Immersed in a Solution of Macromolecules

The Journal of Chemical Physics 22, 1255 (1954); https://doi.org/10.1063/1.1740347

Colloids dispersed in polymer solutions. A computer simulation study

The Journal of Chemical Physics 100, 6873 (1994); https://doi.org/10.1063/1.467003

Theory of the depletion force due to rodlike polymers

The Journal of Chemical Physics 106, 3721 (1997); https://doi.org/10.1063/1.473424

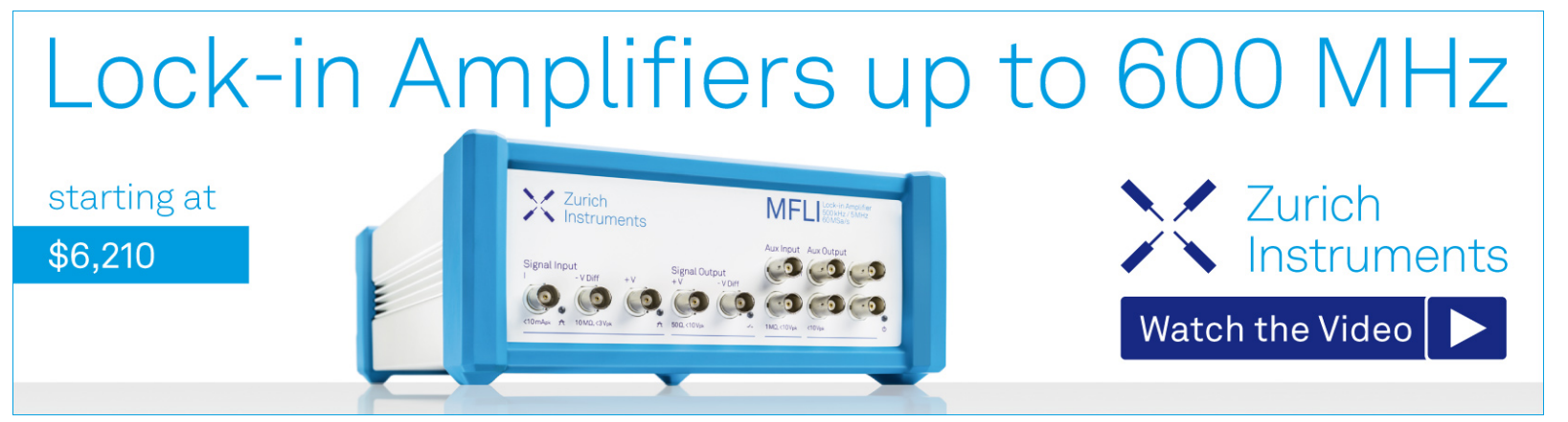

J. Chem. Phys. 117, 5415 (2002); https://doi.org/10.1063/1.1499717

117, 5415

(c) 2002 American Institute of Physics. 


\title{
Polymer depletion interaction between a colloid particle and a wall: A Monte Carlo study
}

\author{
Andrey Milchev ${ }^{\text {a) }}$ \\ Institute for Physical Chemistry, Bulgarian Academy of Sciences, 1113 Sofia, Bulgaria \\ Aniket Bhattacharya ${ }^{\text {b) }}$ \\ Department of Physics, University of Central Florida, Orlando, Florida 32816
}

(Received 25 April 2002; accepted 19 June 2002)

\begin{abstract}
An off-lattice bead-spring model of a polymer solution in a container with impenetrable walls is used to study the depletion interaction of a colloid particle with the planar wall by means of a Monte Carlo simulation. As expected, this interaction is found to depend essentially on the ratio $\rho$ $=R / R_{g}$ of the particle radius $R$ to the mean radius of gyration $R_{g}$ of the polymer chains in the case of dilute and semidilute solutions. For large particle to polymer size ratio $\rho>1$ this effective force is attractive and decreases steadily with growing distance $D$ of the colloid from the wall. It is found to scale linearly with $\rho$ in agreement with recent theoretical predictions. In the opposite case of $\rho<1$ the depletion force is found to change nonmonotonically with $D$ and go through a maximum at a particular distance $D_{\max } \leqslant R_{g}$. In both cases, however, local variations of the polymer density profile, which we detect at higher polymer concentrations, are found to influence the depletion force and even to change it locally from attraction to repulsion. The monomer density distribution far away from/or around the colloid in the vicinity of the wall is also investigated and related to the observed behavior of the depletion force. (c) 2002 American Institute of Physics. [DOI: 10.1063/1.1499717]
\end{abstract}

\section{INTRODUCTION}

The polymer-induced depletion interaction between mesoscopic colloid particles in a solution of nonadsorbing polymer chains is of fundamental interest in colloid physics. ${ }^{1}$ For entropic reasons the chains avoid the space between two close particles, or between a particle and a planar wall, and create an effective attraction among the colloid particles, or push the particles toward the walls of the container. This depletion interaction has been used to explain phase diagrams of colloid-polymer mixtures ${ }^{2}$ and is believed to be important for a variety of interesting colloid systems such as casein micelles, ${ }^{3}$ hemoglubine, ${ }^{4}$ and globular proteins. ${ }^{5}$ Not surprisingly, the problem has attracted much scientific attention and has been addressed both theoretically ${ }^{6-10}$ as well as by means of computer simulations. ${ }^{11-14}$ In most of the theoretical work on the depletion interaction one employs the simple representation of the polymer coils as penetrable hard spheres (PHSs) by Asakura and Oosawa. ${ }^{6}$ Dilute or semidilute solutions of nonintersecting polymer chains are mapped onto a fluid of "soft" spheres interacting via a concentrationdependent effective potential ${ }^{13,14}$ although one should be aware that the PHS approach fails if the polymers are much larger than the colloids. Such cases in which a long flexible chain cannot be reduced to a single degree of freedom have been considered using scaling and field theories, ${ }^{15}$ mean field, ${ }^{7}$ and integral equation techniques. ${ }^{16}$ Recently experimental measurements of the effective interaction between two individual particles or for a single particle near a wall have been reported ${ }^{17,18}$ too.

\footnotetext{
${ }^{a)}$ Electronic mail: milchev@ipc.bas.bg

${ }^{b)}$ Electronic mail: aniket@ physics.ucf.edu
}

In this paper we investigate the depletion interaction between a spherical particle and a planar wall in dilute and semidilute monodisperse solutions of free flexible polymer chains using an off-lattice Monte Carlo bead-spring model. Earlier the problem was treated theoretically by Bringer et al., ${ }^{8}$ who based their treatment on the well-known diffusion equation satisfied by the partition function of a random walk, or, alternatively, on minimization of a GinzburgLandau functional. In the case of ideal chains they derived a number of analytic results demonstrating that the depletion interaction depends crucially on the polymer to particle size ratio. In contrast, in the present simulational study the excluded volume interactions of the polymers are inherent in the model which also takes into account all the fluctuations which are neglected as a rule in the analytic mean-field like treatments.

The paper is organized as follows: In Sec. II we give a brief summary of theoretic results pertaining to limiting cases where a number of analytic expressions ${ }^{8}$ for RW are available. The model used in the simulations is introduced in Sec. III. In Sec. IV we discuss the polymer density profiles at the container walls and around the colloid particle, and in Sec. V present our findings about the polymer-induced depletion force on a sphere for the cases of large and small sphere to polymer size ratio. We end this report with a brief summary of our conclusions in Sec. VI.

\section{SUMMARY OF SCALING PREDICTIONS}

For a spherical colloid particle of radius $R$ which is immersed in a dilute solution of polymer chains of mean gyration radius $R_{g}$ and kept at a distance $D$ apart from a planar wall the depletion interaction attains a universal form when 
the three lengths, $R, D, R_{g}$ are much larger than microscopic lengths and persistent length characterizing the degree of stiffness of the chain. ${ }^{8}$ The free energy of polymer-induced interaction is proportional to the number density $n_{b}$ of chains in the bulk and is independent of the microscopic lengths scaling (in terms of the ratios $\zeta=D / R_{g}, \rho=R / R_{G}$ ) as

$$
\mathcal{G}=p R^{d} Y\left(D / R_{g}, R / R_{g}\right)
$$

with $p=n_{b} k_{B} T$ the bulk osmotic pressure of the chains, $d$ the dimensionality, and $Y(\zeta, \rho)$ a universal scaling function.

Generally for chains with excluded volume interactions one has for the case of large particles: ${ }^{10}$

$$
\mathcal{G} \rightarrow p R^{(d-1) / 2} R_{g}^{(d+1) / 2} \mathcal{Y}(\zeta), \quad \zeta \ll \rho
$$

with a scaling function $\mathcal{Y}$ yielding a polymer-induced attractive force $\partial \mathcal{G} / \partial \zeta$ which decreases monotonically with the distance $\zeta$ from the wall. This behavior is in qualitative agreement with the PHS-approximation of Asakura and Oosawa. ${ }^{6}$ In contrast, for small colloid particles one finds ${ }^{9}$

$$
\mathcal{G} \rightarrow p R^{d-(1 / \nu)} R_{g}^{1 / \nu} \widetilde{\mathcal{Y}}(\zeta), \quad \rho \ll \zeta
$$

with a scaling function $\tilde{\mathcal{Y}}=A\left[\Phi_{h}(\zeta)\right]$ where $\nu \approx 0.589$ is the Flory exponent, $A$ is a known universal number, ${ }^{10}$ and $\Phi_{h}$, normalized to $\Phi_{h}(\infty)=1$, is the monomer density at distance $\zeta$ from the wall if the particle is absent. Since $\Phi_{h}$, which increases steadily with $\zeta$, has a point of inflection, the force $\partial \mathcal{G} / \partial \zeta$ should have a maximum at certain $\zeta_{\max } \approx 1$ and increase for $0<\zeta<1$.

For Gaussian chains $\mathcal{G}=p R R_{g}^{2} \mathcal{Y}(\zeta, \rho)$ and simple explicit expressions can be obtained analytically in several limiting cases: ${ }^{8,19}$ For small distances from the wall the forces on large and small colloid particles albeit attractive, behave differently, that is, initially they decrease or increase with distance $\zeta$ :

$$
\mathcal{Y}(\zeta, \infty) \rightarrow-4 \pi \ln 2+4 \sqrt{2 \pi} \zeta-\pi \zeta^{2}, \quad \zeta \rightarrow 0
$$

and

$$
\mathcal{Y}(\zeta, 0) \rightarrow-2 \pi+4 \pi \zeta^{2}, \quad \zeta \rightarrow 0 .
$$

At larger distances $\zeta$, however, both expressions merge, the forces $\partial \mathcal{Y} / \partial \zeta$ vanish much in the same way independent of $\rho$ :

$$
\mathcal{Y}(\zeta, \rho) \rightarrow-\frac{8 \sqrt{2 \pi}}{\zeta^{3}} \exp \left(-\frac{\zeta^{2}}{2}\right), \quad \zeta \rightarrow \infty,
$$

and one can show ${ }^{8}$ that even the values $\mathcal{Y}(\zeta=1, \rho)$ for different $\rho$ 's should be very close to one another.

\section{MODEL AND SIMULATION TECHNIQUE}

The off-lattice bead-spring model has been used previously for simulations of polymers both in the bulk ${ }^{20,21}$ and near confining surfaces, ${ }^{22-26}$ therefore we describe here the salient features only. Each polymer chain contains $N$ effective monomers connected by anharmonic springs described by the finitely extendible nonlinear elastic (FENE) potential,

$$
U_{\mathrm{FENE}}=-\frac{K}{2} R^{2} \ln \left[1-\frac{\left(l-l_{0}\right)^{2}}{R^{2}}\right] .
$$

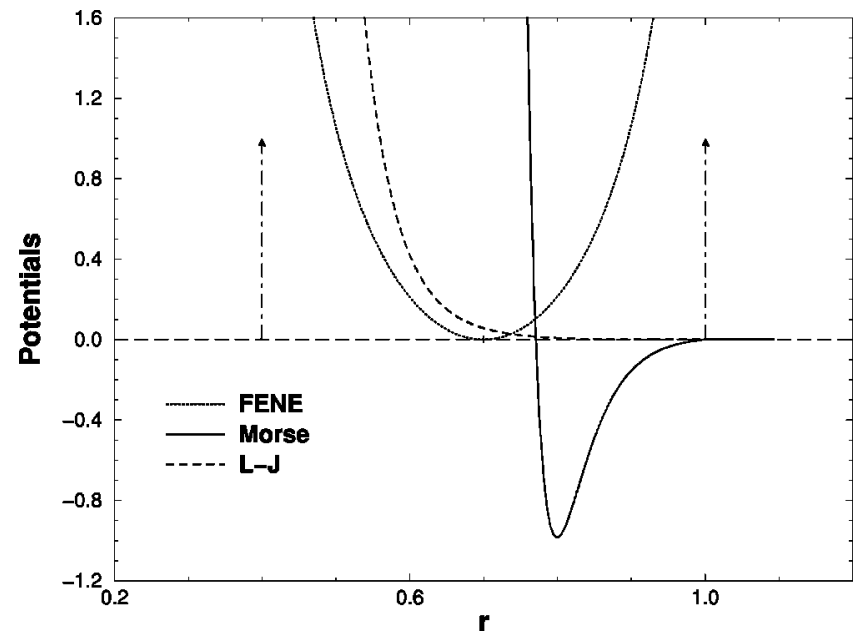

FIG. 1. Plot of the bonded (FENE), Eq. (7)—dotted line, and nonbonded (Morse), Eq. (8) - full line, potentials used for the monomer-monomer interactions in the simulation. Vertical arrows mark the minimal, $l_{\min }$ and maximal $l_{\max }$ extension of a polymer bond. The colloid particle acts on the polymers by means of truncated and shifted Lennard-Jones (LJ) potential, Eq. (9)—dashed line, of which only the repulsive branch is retained.

Here $l$ is the length of an effective bond, which can vary in between $l_{\min }<l<l_{\max }$, with $l_{\min }=0.4, l_{\max }=1$ being the unit of length, and has the equilibrium value $l_{0}=0.7$, while $R=l_{\max }-l_{0}=l_{0}-l_{\min }=0.3$, and the spring constant $K$ is taken as $K / k_{B} T=40$. The nonbonded interactions between the effective monomers are described by the Morse potential-cf. Fig. 1,

$$
U_{M}=\epsilon_{M}\left\{\exp \left[-2 \alpha\left(r-r_{\min }\right)\right]-2 \exp \left[-\alpha\left(r-r_{\min }\right)\right]\right\},
$$

where $r$ is the distance between the beads, and the parameters are chosen as $r_{\min }=0.8, \epsilon_{M}=1$, and $\alpha=24$. Owing to the large value of this latter constant, $U_{M}(r)$ decays to zero very rapidly for $r>r_{\min }$, and is completely negligible for distances larger than unity. This choice of parameters is useful from a computational point of view, since it allows the use of a very efficient link-cell algorithm. ${ }^{27}$ Physically, these potentials Eqs. (7), (8) make sense when one interprets the effective bonds as kinds of Kuhn segments, comprising a number of chemical monomers along the chain, and thus the length unit $l_{\max }=1$ corresponds physically to $1 \mathrm{~nm}$ rather than to the length of a covalent $C-C$ bond (which would only be about $1.5 \AA$ ). Therefore, it also makes sense to treat the surface of the impenetrable container walls on this coarse-grained length scale as perfectly flat and smooth: Any atomistic corrugation of the plane surface would be on a scale much finer than the length scale $l_{\max }$ of the Kuhn segment, and hence is not resolved in our coarse-grained model. Since for this model the theta temperature has been found as $k_{B} \Theta \approx 0.62,{ }^{21}$ working at temperature $T=1$ keeps our polymer solution in the "good solvent" regime.

The colloid particles are taken as spherically shaped and are characterized by their radius $R$, and the distance $D$ between their surface and the hard wall at the bottom of the container $(z=0)$. In order to measure the force, exerted by the polymers on the colloid particle, we now assume that the interaction between the sphere and the effective monomers at 


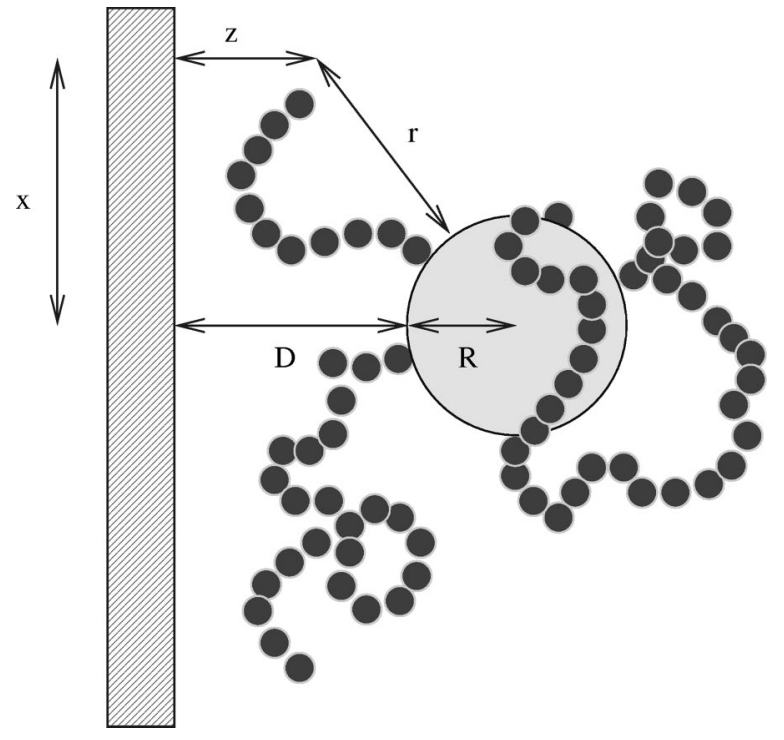

FIG. 2. The colloid is shown schematically as a spherical particle of radius $R$ embedded in a polymer solution at distance $D$ of a planar wall.

distance $r$ from the colloid surface is given by the (shifted) repulsive branch of the Lennard Jones potential,

$$
V_{\text {coll }}(r)=\left\{\begin{array}{l}
4\left[\left(\frac{\sigma}{R-r}\right)^{12}-\left(\frac{\sigma}{R-r}\right)^{6}\right]+1, \quad R<r<\sigma \\
0, \quad r \geqslant \sigma,
\end{array}\right.
$$

where the range parameter $\sigma=1$ for the Lennard-Jones potential. Since $V_{\text {coll }}(r=R)=\infty$, one should bear in mind that the real effective radius of the colloid particles in our study exceeds the sphere radius $R$ so that at temperature $T=1$ the spheres repulse polymers already at distances $\approx R$ $+0.558 \sigma$. This correction has been taken into account in the analysis of our results. One should note also that the total volume available to the polymer is reduced by the presence of a colloid particle. For example, this correction yields the value of 0.127 instead of $\phi=0.125$ for the number density.

Most of the simulations have been carried out for polymer chains of length $N=32$ and mean radius of gyration $R_{g} \approx 2.47$, or with $N=256, R_{g} \approx 8.48$ at monomer densities $\phi=0.0625,0.125$, although some results were obtained for $N=512$, too. Both the polymer chains as well as the sphere which represents a colloid particle are placed in a cubic box of linear size $L, L=32$, or $L=64$, with impenetrable walls at $z=0$ and $z=L$, and periodic boundary conditions in $x, y$ direction-Fig. 2. After initial equilibration of the system, measurements of the average force $F$ acting on the colloid are taken over 60 runs each of length $1 \times 10^{6}$ Monte Carlo steps (MCS) whereby at each MCS all polymer beads are picked at random and randomly displaced at distances $-0.5 \leqslant \Delta x, \Delta y, \Delta z \leqslant 0.5$. Apart from the force $F$, exerted on the spherical particle, we also determine the density profiles close and far away from the colloid as well as a number of properties which characterize the polymer system as mean chain end-to-end distance, radius of gyration $R_{g}$ and mean squared displacements of the chains. Since the relaxation of long chains, i.e., their diffusive displacement at distances of the order of their size, $R_{g}$, takes typically times of the order

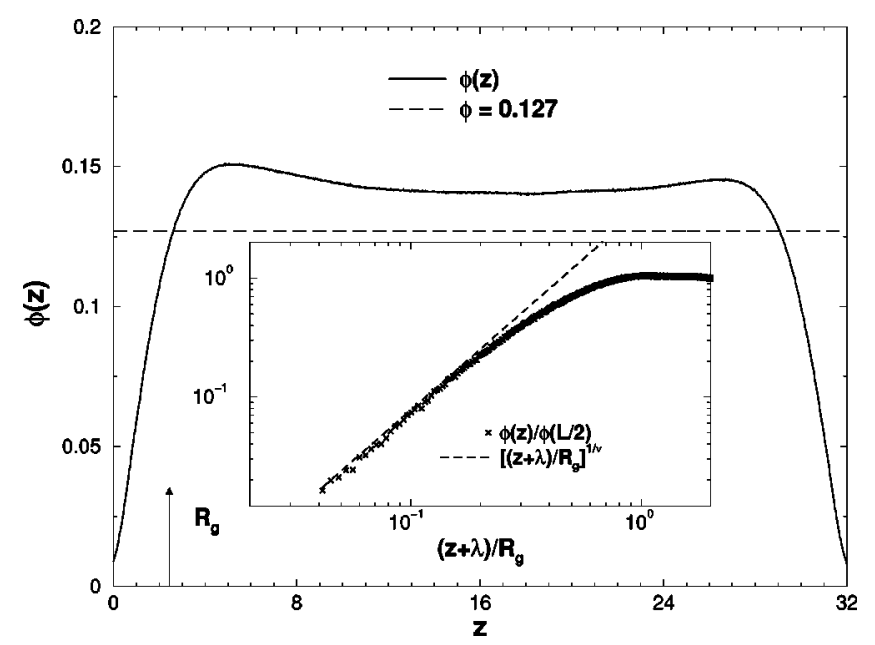

FIG. 3. Density profile $\phi(z)$ (full line) between the hard walls far from the colloid particle for chains with length $N=32$ and total density (without colloid particle) $\phi=0.125$ (dashed line) in a box of size $L=32$. The inset demonstrates in a $\log -\log$ plot that $\phi(z)$, normalized by the density $\phi(L / 2)$ in the middle of the box, scales as expected with power $\nu^{-1}$ against the distance from the wall $z+\lambda$ where the extrapolation length $\lambda=0.35$. Here $N=256, R_{g}=8.48$, and $\phi=0.0625$.

of $\tau \propto N^{2 \nu+1}$ with $\nu \approx 0.6$, it is clear that the exploration of small particle to polymer size cases becomes rapidly difficult with growing chain length $N$ and the statistical accuracy of such results poses problems.

\section{POLYMER DENSITY PROFILES AT THE WALL}

Figure 3 shows the monomer density profile $\phi(z)$ of the polymer solution perpendicular to the container walls for chains with length $N=32$ at total number density $\phi=0.125$ far away from the sphere representing the colloid particle in the system. For entropic reasons the chains tend to stay away from the walls and one observes a steep increase of the monomer density over intervals of the order of the polymer size $R_{g} \approx 2.48$ close to the walls before $\phi(z)$ levels off to a bulk value. Evidently, for $\phi=0.125$ the density profile reveals two relatively small maxima at distance $z \approx 2 R_{g}, L$ $-2 R_{g}$ which are slightly above the density in the bulk. This feature indicates that the concentration $\phi=0.125$ is close to the upper bound of the dilute regime and is probably responsible for the slight overshoot in the depletion force on the colloid which we measure at these distances (see Sec. V). Thus, it appears that, depending on the particular density profile established for a given $\phi$, the (generally) attractive depletion force may change to slight repulsion at certain distances where the density profile changes are not monotonic. If much lower concentration is taken, however, the density profile increases steadily with $z$ and becomes perfectly flat at distances $z \geqslant R_{g}$, as shown in the inset of Fig. 3 where we deal with chains of $N=256$ at $\phi=0.0625$. Anticipating, we note that in this case the resulting depletion force on the particle decreases monotonically to zero (see the following) and shows no overshoots.

In agreement with relation, derived earlier by Eisenriegler $^{19}$ and confirmed later by computer 

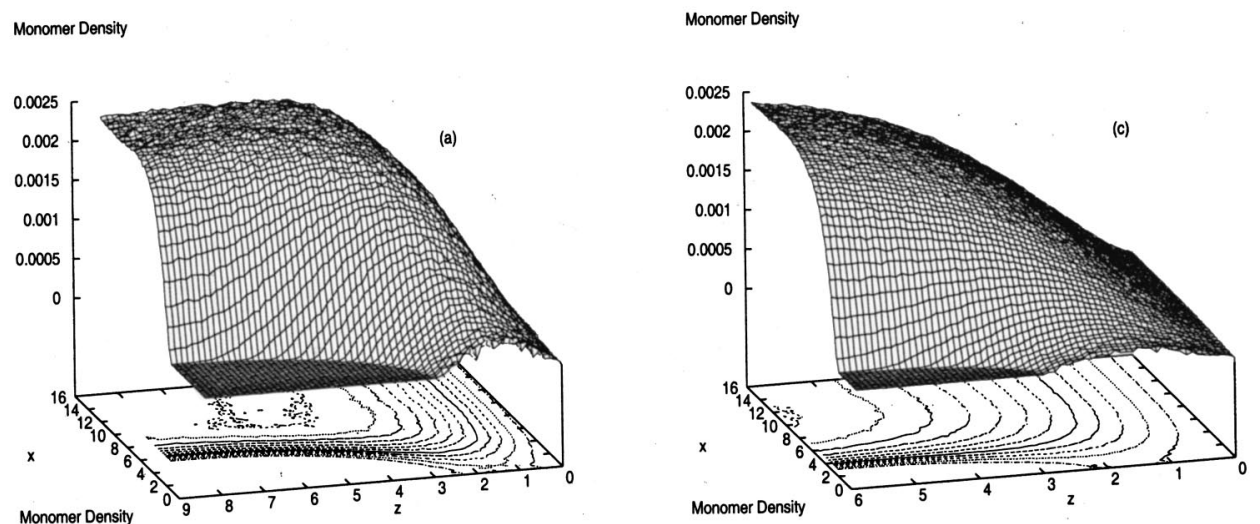

FIG. 4. Monomer density distribution $\Phi(\mathbf{r})$ around a colloid particle. For symmetry reasons only a section of the box: $0 \leqslant z \leqslant D+R, 0 \leqslant x, y \leqslant L / 2$ is shown. (a) Large particles: $N=32, R$ $=5, D=3.5, \phi=0.125$. (b) The same as in (a) for $D=11.0$. (c) The same as in (a) for small particles: $N=256$ and
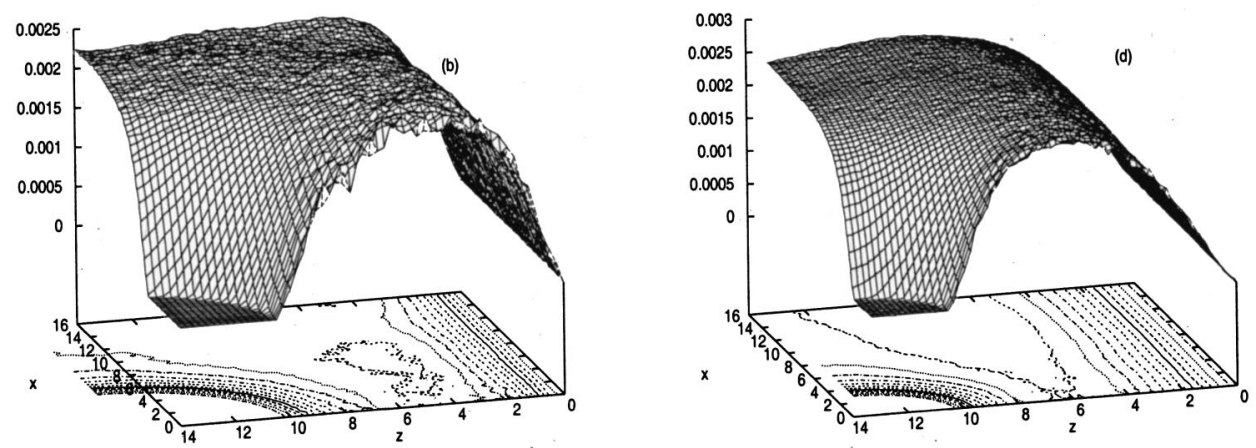
$R=2.5$. (d) The same as in (c) for $D$ $=11.0$. The contour iso-density lines separate the whole interval of density variation into 12 sub-intervals.

simulations, ${ }^{28}$ one finds that the density scales with $z$ as a power law with exponent $1 / \nu$, that is, $\phi(z) \propto(z+\lambda)^{1 / \nu}$, where we use as usual the concept of an extrapolation length $^{28} \lambda$ (this microscopic length is of the order of the monomer radius, $\lambda \approx 0.35$ ) so as to take into account effects of discreteness which are ignored in the theoretical treatment.

While we previously presented monomer density profiles undisturbed by the colloid, in Fig. 4 we show the density distribution in the immediate vicinity of the spherical particle and close to the planar wall. Due to symmetry considerations, the three-dimensional plots cover only a quarter of the container which in the $z$ direction is limited between the wall and the center of the sphere whereas in $x$ (or, equivalently, in the $y$ direction) it goes from the projection of the particle center up to the end of the box at distance $L / 2$. Figures 4(a) and 4(b) correspond to the case of large particles (short chains), $\rho \approx 2$, while Figs. 4(c) and 4(d) refer to small particles (much longer chains) with $\rho \approx 0.3$. Not surprisingly, at the closest distance between the sphere and the wall in the case of $D=3.5$-cf. Figs. 4(a) and 4(c) — the density $\Phi(\mathbf{r})$ is considerably larger for shorter chains, Fig. 4(a), than for the longer ones. At larger distance $D=11.0$ one may observe the formation of a local density maximum between the particle and the wall [see the highest contour line of constant density in Fig. 4(b)] which even exceeds the shallow maxima of the undisturbed distribution far from the sphere, mentioned previously in connection with Fig. 3. Clearly, this local accumulation of density is expected to repel and push the colloid away from the wall and into the bulk, an effect which is probably due to the presence of excluded volume interactions in the simulation and was not reported by Bringer et al. ${ }^{8} \mathrm{~A}$ closer inspection and comparison of the contour lines in Figs. 4(c) and 4(d) reveals also that for $x=0$ the density increase around the colloid is much steeper for longer chains than for short ones, in contrast to the increase in the undisturbed density profiles at the wall, thus demonstrating that the sphere partially penetrates into the larger polymer coils.

\section{POLYMER DEPLETION FORCE}

One of the main results of our investigation is shown in Fig. 5, which displays the variation of the polymer-induced

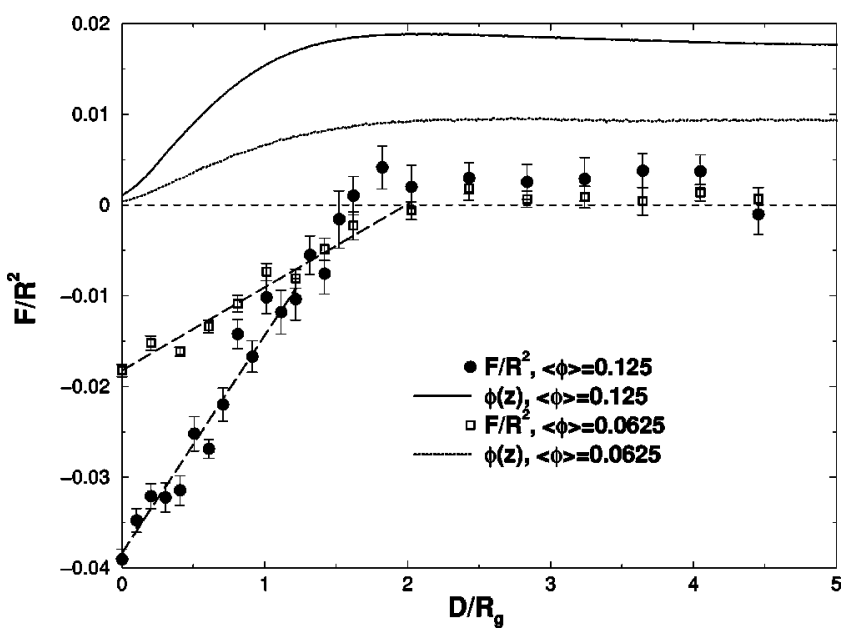

FIG. 5. Variation of the polymer-induced pressure exerted on a large colloid particle ( $\rho \approx 2$ ) with radius $R=5.0$ at mean monomer density $\phi=0.125(\bullet)$ and $\phi=0.625(\square)$ with dimensionless distance $\zeta=D / R_{g}$ from the container wall. The mean size of the polymer chains here is $R_{g} \approx 2.48$. The density profiles in the vicinity of the wall are also shown. Linear dashed lines are guides for the eye. 


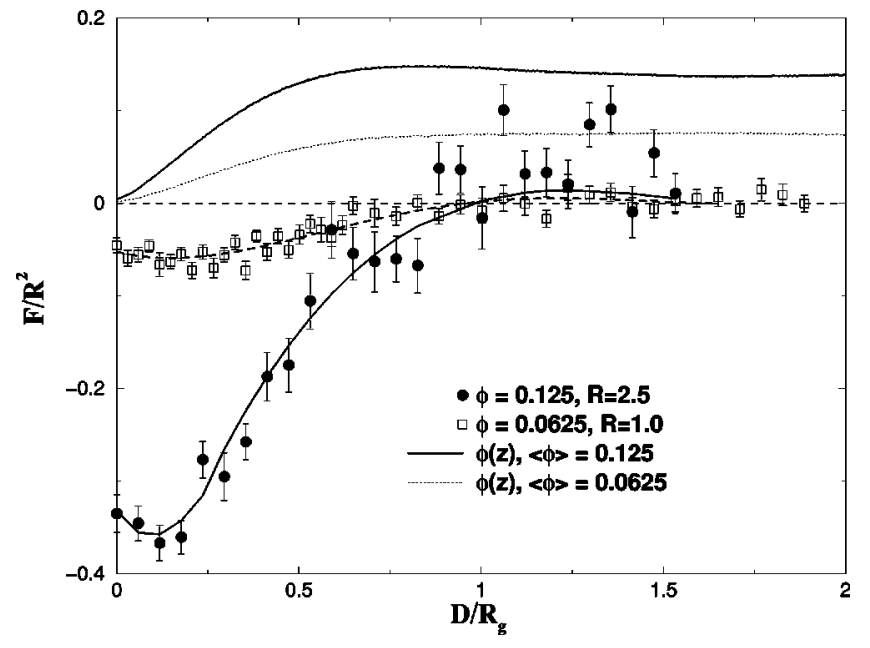

FIG. 6. The same as in Fig. 5 in the case of small colloids with $\rho \approx 0.3$ at $\phi=0.125$, and $\rho \approx 0.118$ at $\phi=0.0625$. Polynomial regression lines are drawn through the data points as guide for the eye.

pressure on a big colloid particle $\left(\rho=R / R_{g} \approx 2\right)$ with the dimensionless distance from the wall $\zeta=D / R_{g}$ along with the respective density profiles $\phi(\zeta)$ at the wall for $\phi$ $=0.125$ and $\phi=0.0625$. Although the measurements bear considerable error bars, one can clearly verify that in both cases the depletion force is predominantly attractive and vanishes at distances $\zeta \approx 2$. The correlation between depletion force and density profile, indicated in Sec. IV, is evident. The pressure, driving the particle in direction of the nearby wall, decreases steadily to zero for $\phi=0.0625$ when the density profile gradually reaches saturation at bulk values for $\zeta \approx 2$. At twice higher overall density, $\phi=0.125$, this pressure doubles, in accord with Eq. (2), and even changes at $\zeta$ $\approx 1.5$ in direction away from the wall, reflecting a slight overshoot in the corresponding density profile. Also in agreement with predictions, ${ }^{8}$ the initial variation of the depletion force $F / R^{2}$ is linear with $\zeta$, as a first derivative, $\partial \mathcal{Y} / \partial D$, of the scaling function in Eq. (4) would result in. We emphasize the change in sign and direction of the polymer-induced depletion force, observed in our simulation at the higher polymer concentration, because it could be viewed as an indication that at even higher concentrations, when the density profile at the walls is characterized by well expressed oscillations, ${ }^{25}$ the resulting depletion force might attain more complicated form and create a kind of stratified structure of a colloid at the vicinity of the planar boundaries.

In the opposite case of colloid radii $R$ smaller than the mean size $R_{g}$ of the polymer chains, shown in Fig. 6, the variation of force with distance appears similar, although an additional shallow minimum (i.e., a maximum of the force in absolute terms) at distances close to the wall appears to be visible despite the statistical uncertainty. This nonmonotonic variation of the force with $\zeta$ has been predicted ${ }^{8}$ for the case of small colloid to chain ratio $\rho$ (here $\rho=R / R_{g}$ $\approx 0.118,0.295$ ), too [cf. Eq. (3) and the following text], and in this aspect the behavior shown in Fig. 6 is clearly different from that in Fig. 5. One should note, however, that from simulational point of view this case of $\rho \ll 1$ is much more

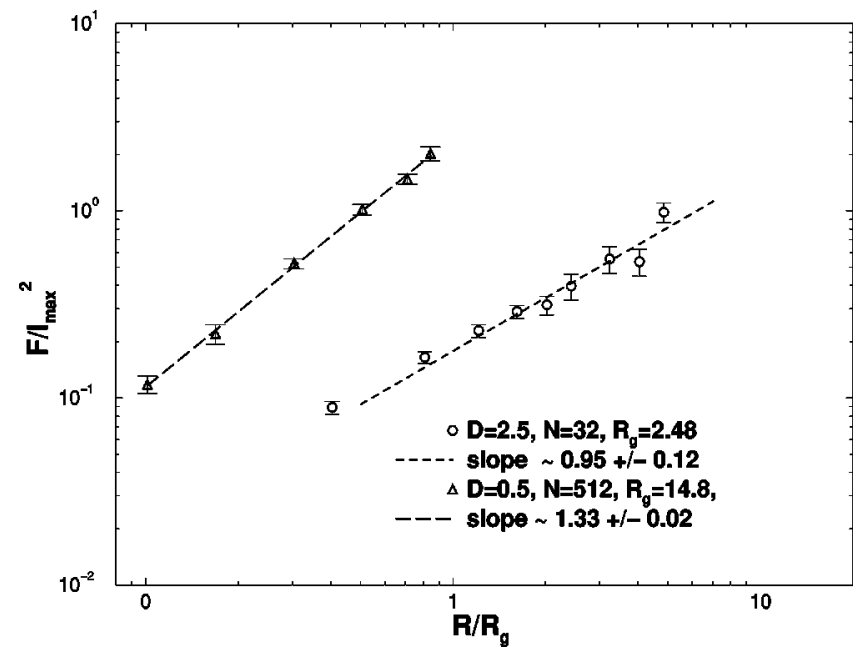

FIG. 7. Scaling of the total depletion force acting on a colloid particle with its radius $R$ for large $\bigcirc$ and small $\triangle$ ratio $\rho=R / R_{g}$.

demanding and the large statistical errors allow here only qualitative conclusions.

Finally, Fig. 7 presents the observed scaling of the depletion force with changing colloid to polymer size ratio $\rho$. The measured pressure on the spherical particle grows linearly with particle radius $R$ in the case of large particles, that is, within statistical error we find an exponent of $0.95 \pm 0.12$ against the expected value of $(d-1) / 2=1-$ cf. Eq. (2), while for small particles we determine a power of 1.33 \pm 0.03 which is very close to $d-1 / \nu$, predicted by Eq. (3). Nevertheless, one should view these findings with caution because $\rho$ varies in both cases in comparatively narrow intervals, and the statistical uncertainty is considerable.

\section{CONCLUSIONS}

We have investigated the polymer-induced depletion interaction between a spherical particle and the planar boundary wall in a dilute solution of flexible nonadsorbing polymer chains with excluded volume interactions in the good solvent regime. We find good qualitative agreement with earlier theoretical predictions ${ }^{8}$ pertaining to the depletion force on the colloid in the vicinity of a wall:

(i) for large particles this attractive force goes steadily to zero over distances $\approx 2 R_{g}$ from the planar boundary whereby the variation of $F$ with distance $\zeta$ for $\zeta \leqslant 1$ is linear, as predicted, and

(ii) for small particles $F$ changes nonmonotonically with $\zeta$, going through a maximum at $\zeta_{\max } \approx 1$, in agreement with expectations, ${ }^{8}$ too.

An immediate conclusion from these findings would be a possible segregation of colloids at the container walls in a polydisperse system in which the variance of particle to polymer size ratio $\rho=R / R_{g}$ is sufficiently large. But even in a monodisperse colloid system it may happen, depending on the particular density profile of the polymer solution at the wall, that depletion forces may change their sign and repel particles from the container walls rather than attracting them-our simulational results support this conclusion for 
both large and small particle to polymer size ratio $\rho$. Thus, apart from the generally attractive nature of the polymerinduced colloid-wall interactions, a considerably richer behavior appears possible in principle. Clearly, considerably more investigations are needed in order to reach a comprehensive picture of polymer-induced interactions, and the present simulation may be considered as a step in this direction only. Thus one should be aware of the fact, of course, that analytic results envisage predominantly asymptotic regimes which are difficult to test directly in computer simulation. Despite rather involved computations, the statistical accuracy of our computer experiments is in many cases not sufficient for precise quantitative comparison and they can be viewed as providing qualitative indications only. Nevertheless we believe that our study corroborates the present understanding of polymer-induced interactions in mesoscopic systems and will be followed by further extensive investigations in the field.

\section{ACKNOWLEDGMENTS}

This work was supported by a National Science Foundation (ENG/ECS and CISE/EIA) Grant No. 0103587 (A.B.). One of us, A. M., acknowledges the support and hospitality of the UCF, Orlando, FL during this study. He is also indebted to P. Grassberger and A. Bringer from the FZ, Jülich, Germany, for an invitation to visit the Forschungszentrum and for useful discussions.

${ }^{1}$ Colloid Physics, Proceedings of the Workshop on Colloid Physics, University of Konstanz, Germany, 1995 [Physica A 235, 120 (1997)].

${ }^{2}$ H. N. W. Lekkerkerker, Physica A 213, 18 (1995).

${ }^{3}$ R. Tuinier, E. ten Grotenhuis, C. Holt, P. A. Timmins, and C. G. de Kruif, Phys. Rev. E 60, 848 (1999).
${ }^{4}$ J. Janzen and D. E. Brooks, Clin. Hemorheol. 9, 695 (1989).

${ }^{5}$ A. M. Kulkarni, A. P. Chattarjee, K. S. Schweizer, and C. F. Zukoski, Phys. Rev. Lett. 85, 4554 (1999).

${ }^{6}$ S. Asakura and F. Oosawa, J. Chem. Phys. 22, 155 (1954).

${ }^{7}$ J. F. Joanny, L. Leibler, and P. G. de Gennes, J. Polym. Sci., Polym. Phys. Ed 17, 1073 (1979).

${ }^{8}$ A. Bringer, E. Eisenriegler, F. Schlesener, and A. Hanke, Eur. Phys. J. B 11, 101 (1999).

${ }^{9}$ E. Eisenriegler, J. Chem. Phys. 113, 5091 (2000); J. Phys.: Condens. Matter 12, A227 (2000)

${ }^{10}$ A. Hanke, E. Eisenriegler, and S. Dietrich, Phys. Rev. E 59, 6853 (1999).

${ }^{11}$ E. Meijer and D. Frenkel, J. Chem. Phys. 100, 6873 (1994).

${ }^{12}$ R. Tuinier, G. Vliegenthart, and H. Lekkerkerker, J. Chem. Phys. 113, 10768 (2000).

${ }^{13}$ A. A. Louis, P. G. Bolhuis, J. P. Hansen, and E. J. Meijer, Phys. Rev. Lett. 85, $2522(2000)$.

${ }^{14}$ P. G. Bolhuis, A. A. Luis, and J. P. Hansen, J. Chem. Phys. 114, 4296 (2001).

${ }^{15}$ E. Eisenriegler, A. Hanke, and S. Dietrich, Phys. Rev. E 54, 1134 (1996).

${ }^{16}$ A. P. Chatterjee and K. S. Schweizer, J. Chem. Phys. 109, 10477 (1998).

${ }^{17}$ R. Verma, J. C. Crocker, T. C. Lubensky, and A. G. Yodh, Phys. Rev. Lett. 81, 4004 (1998).

${ }^{18}$ D. Rudhardt, C. Bechinger, and P. Leiderer, Phys. Rev. Lett. 81, 1330 (1998).

${ }^{19}$ E. Eisenriegler, Phys. Rev. E 55, 3116 (1997).

${ }^{20}$ A. Milchev, W. Paul, and K. Binder, J. Chem. Phys. 99, 4786 (1993).

${ }^{21}$ A. Milchev and K. Binder, Macromol. Theory Simul. 3, 915 (1994).

${ }^{22}$ A. Milchev and K. Binder, J. Chem. Phys. 114, 8610 (2001).

${ }^{23}$ A. Milchev and K. Binder, Macromolecules 29, 343 (1996).

${ }^{24}$ A. Milchev and K. Binder, J. Phys. II 6, 21 (1996); Eur. Phys. J. B 9, 477 (1998).

${ }^{25}$ R. B. Pandey, A. Milchev, and K. Binder, Macromolecules 30, 1194 (1997).

${ }^{26}$ A. Milchev and K. Binder, J. Chem. Phys. 106, 1978 (1997).

${ }^{27}$ I. Gerroff, A. Milchev, W. Paul, and K. Binder, J. Chem. Phys. 98, 6526 (1993).

${ }^{28}$ A. Milchev and K. Binder, Eur. Phys. J. B 3, 477 (1998). 